ISSN: 2386-3919 - e-ISSN: 2386-3927

DOI: http://dx.doi.org/10.14201/et20163413555

\title{
EL DESARROLLO DE LA IDENTIDAD PROFESIONAL DOCENTE EN LA FORMACIÓN INICIAL DEL PROFESORADO DE SECUNDARIA
}

\section{The development of the Teaching Professional Identity in the initial training of secondary teachers}

\author{
Rocío Serrano Rodríguez* y Alfonso Pontes Pedrajas** \\ * Departamento de Educación. Facultad de Ciencias de la Educación. \\ Universidad de Córdoba. Correo-e: rocio.serrano@uco.es \\ ** Escuela Politécnica Superior. Universidad de Córdoba.Correo-e: apontes@uco.es
}

Recibido: 13-08-2015; Aceptado: 24-09-2015; Publicado: 30-05-2016

BIBLID [2386-3919 (2016) 34, 1; 35-55]

Ref. Bibl. ROCÍO SERRANO RODRÍGUEZ y ALFONSO PONTES PEDRAJAS. El desarrollo de la Identidad Profesional Docente en la formación inicial del profesorado de secundaria. Enseñanza \& Teaching, 34, 1-2016, 35-55.

RESUMEN: Presentamos un estudio destinado a conocer cómo comienza a construir la Identidad Profesional Docente el alumnado del Máster de Formación del Profesorado de Enseñanza Secundaria y cuáles son las principales implicaciones de este proceso de identificación en la mejora formativa. Mediante un cuestionario abierto, se han explorado las opiniones de 335 estudiantes de la Universidad de Córdoba. Entre las conclusiones destacamos: a) tales estudiantes presentan una Identidad Profesional poco elaborada, apreciándose notables diferencias sobre este tema respecto a otros colectivos profesionales; b) es importante desarrollar actividades que permitan reflexionar sobre la naturaleza de la profesión docente mejorando la identificación de los y las estudiantes con las características de dicha profesión. Algunas de las implicaciones que se derivan de esta investigación apuntan a la necesidad de tener en cuenta los resultados obtenidos a la hora de diseñar el proceso de formación inicial de los futuros profesores de Educación Secundaria. Sobre todo, sería necesario incluir en el diseño curricular del nuevo modelo de formación inicial un tratamiento 
ROCÍO SERRANO RODRÍGUEZ Y ALFONSO PONTES PEDRAJAS

EL DESARROLLO DE LA IDENTIDAD PROFESIONAL DOCENTE EN LA FORMACIÓN INICIAL DEL PROFESORADO DE SECUNDARIA

específico del tema de la Identidad Profesional Docente, que debería ir ligado al conocimiento didáctico del contenido y a la adquisición de competencias docentes reales relacionadas con el desarrollo del currículum educativo de cada materia.

Palabras clave: profesorado; educación secundaria; pensamiento inicial del profesorado; actividades de aula; identidad profesional docente.

SUMMARY: We present a study that aims to know how the students of the Master's Degree in Secondary Teacher Training begin to build their Teaching Professional Identity, as well as what the main implications of this identification process are, in order to improve their training. Through an open questionnaire, a total of 355 opinions of students from the University of Córdoba have been analysed. The conclusions obtained highlight: a) the students show a professional identity that is significantly less developed than in other professional groups; b) it is important to develop activities to reflect on the nature of the teaching profession by improving the identification of students with the characteristics of the teaching profession. Some of the implications from this research point to the need to take into consideration the results obtained when designing the process of initial training of future secondary teachers. Particularly, it would be necessary to include the issue of Teaching Professional Identity more specifically in the curriculum of the new model of initial training. This, moreover, should be linked to the pedagogical knowledge of the contents and the acquisition of real teaching skills according to the development of the educational curriculum for each subject.

Key words: teacher; Secondary education; initial thought educational; classroom activities; teaching professional identity.

\section{INTRODUCCIÓN}

Actualmente, la transición hacia el Espacio Europeo de Educación Superior (EEES) ha supuesto el replanteamiento de la concepción que se tenía sobre la docencia (Hernández y Hernández, 2011; Zabalza, 2012). En este sentido, el profesorado se constituye como uno de los principales agentes que pueden hacer posible el cambio educativo (OCDE, 2011), ajustándose a un perfil profesional que le permita responder de forma adecuada a los nuevos retos y demandas que están surgiendo en la nueva sociedad del conocimiento y de la información.

Esta situación actual nos lleva a plantearnos la necesidad de ofrecer una preparación profesional acorde a las demandas docentes actuales. En particular este estudio forma parte de un programa de investigación e innovación educativa orientado a mejorar el proceso de formación inicial del profesorado de secundaria (FIPS) en la Universidad de Córdoba (Serrano, 2013). Durante la transición del modelo anterior de formación docente (Curso para la obtención del Certificado de Aptitud Pedagógica, conocido como CAP) al nuevo modelo formativo, que se ha configurado en torno al Máster de Formación del Profesorado de Enseñanza Secundaria 
ROCÍO SERRANO RODRÍGUEZ Y ALFONSO PONTES PEDRAJAS

EL DESARROLLO DE LA IDENTIDAD PROFESIONAL DOCENTE EN LA FORMACIÓN INICIAL DEL PROFESORADO DE SECUNDARIA

(Máster FPES), hemos contribuido al desarrollo de diversos proyectos centrados en la exploración del pensamiento inicial docente sobre diferentes aspectos como la reflexión sobre el practicum de la formación inicial, las concepciones sobre los procesos educativos o las necesidades formativas del profesorado de este nivel (Pontes y Serrano, 2010). En el desarrollo de tales estudios ha surgido el interés por explorar las motivaciones hacia la profesión docente (Pontes, Ariza, Serrano y Sánchez, 2011) y las creencias sobre el desarrollo profesional (Pontes, Ariza y Del Rey, 2010), lo que ha derivado en el interés por conocer cómo se construye la Identidad Profesional Docente (IPD) entre los futuros profesores y futuras profesoras de Enseñanza Secundaria, contribuyendo al análisis de aspectos que pueden mejorar el proceso de formación inicial docente en el futuro (Serrano, Pontes y Muñoz, 2012).

2. LA FORMACIÓN DEL PROFESORADO, UN ELEMENTO CLAVE PARA EL DESARROLLO DE LA IDENTIDAD PROFESIONAL DOCENTE

El concepto de IPD fue introducido por Erikson en 1980 y aunque son diversas las clasificaciones que encontramos sobre los elementos que conforman la IPD (Larraín, 2003; Bolívar, 2006), de todas ellas podemos extraer dos componentes claves: una dimensión personal, íntima y peculiar que hace al sujeto diferente de los y las demás, y una dimensión social, compartida y en constante interacción con "Otros y otras». En esta dimensión social cobra vital importancia el reconocimiento de cada sujeto en el ejercicio de la profesión docente y el propio reconocimiento social de la profesión. Según Lobato, Fernández, Garmendia y Pérez (2012), este proceso de construcción de la IPD presenta un carácter dinámico, en el que la interacción social con otras personas próximas juega un papel esencial en la evolución de la construcción social de la identidad (Zabalza, 2012).

La IPD del profesorado en formación o en activo se ha investigado con diferentes técnicas, tales como entrevistas (Bolívar, 2006), cuestionarios de diversos tipos (Pontes et al., 2010; Serrano et al., 2012) o redes de ideas y mapas conceptuales (Sweitzer, 2008; Lim, 2011), adoptando en todos los casos un enfoque indagador orientado a activar la reflexión de los participantes en tales estudios acerca de la naturaleza y características de la profesión docente (Kagan, 1992; Briggs, 2007; Álvarez y San Fabián, 2013).

En un estudio longitudinal, desarrollado por Nias (1989), basado en el seguimiento a un conjunto de docentes en su primera década de trabajo, se comprobó que el desarrollo de la IPD cobra sentido en las interacciones con los y las demás durante el ejercicio y el desarrollo de la vida profesional como docente. Por tanto, la IPD se construirá en espacios sociales de interacción, donde la imagen social y la aceptación de uno mismo se va a ir configurando bajo ese reconocimiento social (Bolívar, Fernández y Molina, 2005; Veiravé, Ojeda, Núñez y Delgado, 2006; Day y $\mathrm{Gu}, 2012)$. La IPD no es una cuestión de pertenencia, algo que se tiene o no se tiene. Se trata de un conjunto de necesidades, valores, experiencias, sentimientos 
ROCÍO SERRANO RODRÍGUEZ Y ALFONSO PONTES PEDRAJAS

EL DESARROLLO DE LA IDENTIDAD PROFESIONAL DOCENTE EN LA FORMACIÓN INICIAL DEL PROFESORADO DE SECUNDARIA

y habilidades que se forman a lo largo de la experiencia personal y profesional de cada uno y cada una, creando dicho sentido de identidad (Korthagen, 2004, 2010). Coincidimos con los trabajos de Ávalos, Cavada, Pardo y Sotomayor (2010) en destacar la importancia, en la constitución de la IPD, de los significados y definiciones que dan de su trabajo y la intensidad con la que se sienten comprometidos.

El desarrollo profesional docente ajustado a las nuevas demandas sociales no puede darse si el profesorado no se encuentra motivado, capacitado e implicado en el buen desempeño de su profesión. Sin embargo, los datos recogidos en diversas investigaciones no parecen ser muy optimistas (Torres, 2010). En los últimos tiempos se viene hablando de "crisis de identidad" del docente de secundaria, expresión con la que se quiere indicar la desmotivación y la falta de implicación en el desempeño de las labores docentes (Bolívar, 2009). En una investigación, realizada por Segovia y Barrero (2012), sobre la "crisis de la identidad" en tiempos de cambio, se formula la necesidad de contribuir a la autocomprensión de la profesión docente. Por otro lado, Martín, Conde y Mayor (2014) hablan de «crisis de identidad» en el profesorado novel universitario, basado en la faltad de autoridad y de experiencias para realizar cambios educativos que mejoren los procesos de enseñanza-aprendizaje.

En un estudio realizado por Sepúlveda y Rivas (2000), se analizaron las cinco biografías profesionales de seis profesores y profesoras de Educación Secundaria, encontrando que la enseñanza no era contemplada como una salida o preferencia personal. Cinco de tales docentes admiten que se dedicaron a la enseñanza más bien por obligación que por vocación. Solo en uno de los casos se manifestó este interés profesional desde una vocación temprana. En investigaciones realizadas con alumnado del CAP, esta vocación aparece muy devaluada (García y Martínez, 2001), indicando que el $88,6 \%$ de los encuestados realizaba el citado curso porque se trataba de un requisito para acceder al cuerpo de profesores y profesoras, pero no realmente vocacional. En estudios posteriores, realizados en la Universidad de Córdoba (Córdoba, Ortega y Pontes, 2009; Pontes et al., 2011), el carácter vocacional referido a la profesión docente caracterizaba a cerca de una tercera parte de los aspirantes a ejercer la docencia en educación secundaria. Por el contrario, en una investigación centrada en la discusión sobre la IPD en Chile (Ávalos y Sotomayor, 2012), un número elevado de docentes de enseñanza media relacionaban la identidad profesional con un componente vocacional elevado, motivados por su profesión y por enseñar al alumnado, a pesar de constatar las dificultades que se presentan en esta etapa educativa.

En la investigación desarrollada por Flores y Day (2006), con profesorado principiante en sus dos primeros años de enseñanza, la construcción, desconstrucción y reconstrucción de sus identidades estuvo influenciada por la formación inicial docente recibida. En esta línea, Marcelo y Vaillant (2009) destacan el proceso de instrucción inicial como la etapa clave para formar docentes apasionados por la enseñanza, o bien todo lo contrario, docentes integrados en un sistema reproductivo donde la motivación y la competencias profesionales se pierden (Hong, 2010; 
EL DESARROLLO DE LA IDENTIDAD PROFESIONAL DOCENTE EN LA FORMACIÓN INICIAL DEL PROFESORADO DE SECUNDARIA

Cabrera y Córdoba, 2011). El trabajo realizado por Gewerc (2011) también refleja como la construcción de la IPD comienza a definirse en el proceso de formación inicial, constituyendo el eje central de un proyecto identitario.

Por otro lado, Danielewicz (2001) describe el relato de seis graduados y graduadas universitarias que participan en un programa de formación del profesorado de secundaria de tres años de duración. En este estudio se muestra cómo van configurando la IPD y cómo esta identidad está influenciada por las apreciaciones positivas o negativas que tienen en la formación inicial durante el periodo de practicum (Valle y Manso, 2010; López y Blázquez, 2012). Concluye el estudio resaltando la necesidad de desarrollar programas de formación, donde el profesorado novel se sitúe en el análisis de problemas prácticos ligados a los contextos educativos actuales (Nilsson, 2008; Marcelo, 2009).

En otros estudios centrados en las opiniones sobre la formación inicial y la profesión docente (Mosquera, 2011) se detectaron concepciones próximas a una visión tradicional de la enseñanza y se exploraron las demandas formativas más relevantes para el ejercicio de la profesión docente, destacando la necesidad de adquirir técnicas de comunicación para mejorar la función docente, lo cual es lógico si se aspira sólo a ser un buen transmisor del conocimiento. En la investigación de Camacho y Padrón (2006), los participantes en un curso de formación inicial relacionaban sus principales inquietudes con el manejo de recursos didácticos variados y el aprendizaje de nuevas metodologías. El alumnado encuestado destacó como elemento esencial de la formación pedagógica recibida la adquisición de técnicas de comunicación y el desarrollo de estrategias de motivación para el alumnado, en coincidencia con otros estudios (Márquez, 2009; Pérez, 2010; Pontes y Serrano, 2010).

Por último, en un estudio más reciente realizado sobre la representación de la IPD de futuros profesores y profesoras, destinado a explorar la imagen o el perfil ideal del docente (Ibarras, 2014), se destaca la necesidad de desarrollar tres aspectos importantes: valores profesionales (tales como respeto, compromiso y responsabilidad), valores o cualidades docentes relacionados con la actividad pedagógica y las relaciones sociales en el aula (tolerancia, solidaridad, empatía) y competencias relacionadas con la formación disciplinar y científica (conocimiento y actualización).

\section{DisEÑO DE LA INVESTIGACIÓN}

Este estudio forma parte de un proyecto de investigación más amplio, relacionado con la exploración del pensamiento inicial de los alumnos y alumnas del Máster FPES en torno a la construcción de la IPD, las necesidades formativas más relevantes para el desarrollo profesional y su relación con las competencias docentes deseables para ejercer la profesión docente en centros de enseñanza secundaria (Serrano, 2013). Los datos recogidos en este proyecto surgen a partir del análisis de documentos escritos por los estudiantes del máster en el desarrollo de actividades 
ROCÍO SERRANO RODRÍGUEZ Y ALFONSO PONTES PEDRAJAS EL DESARROLLO DE LA IDENTIDAD PROFESIONAL DOCENTE EN LA FORMACIÓN INICIAL DEL PROFESORADO DE SECUNDARIA

de enseñanza-aprendizaje realizadas en el aula. Por limitación de espacio en este estudio nos vamos a centrar en analizar las respuestas a un conjunto de cuestiones abiertas sobre la IPD, que los profesores y las profesoras en formación han trabajado en el aula durante el desarrollo de una clase del Máster FPEs. Tras realizar un estudio piloto de las concepciones personales sobre la IPD, por parte de una muestra reducida de estudiantes del área de ciencia y tecnología (Serrano et al., 2012), se observó que las ideas previas de los futuros y futuras docentes sobre esta temática eran suficientemente interesantes para llevar a cabo un estudio más amplio, cuyos resultados se muestran en este trabajo.

\subsection{Enfoque}

En esta investigación se ha adoptado un enfoque metodológico mixto, basado en un análisis cualitativo y cuantitativo de los datos recogidos. En primer lugar intentamos acercarnos al conocimiento de la realidad social analizando cualitativamente las opiniones del alumnado y en segundo lugar se realiza un análisis cuantitativo de frecuencias y porcentajes correspondientes a las diversas categorías de ideas recogidas en la investigación. Se trata, por tanto, de un estudio descriptivo, basado en un diseño no experimental.

\subsection{Objetivos}

La finalidad global de la presente investigación ha sido conocer de qué forma comienzan a construir la Identidad Profesional Docente los estudiantes del Máster de Formación del Profesorado de Enseñanza Secundaria y qué implicaciones se derivan de este conocimiento para mejorar el proceso de formación.

De manera más concreta, se establecieron los siguientes objetivos específicos relacionados con el alumnado del Máster FPES:

a) Explorar sus concepciones previas sobre la noción de IPD y las características que engloba este concepto.

b) Analizar cuándo y cómo comienza el desarrollo de la IPD entre los futuros y futuras docentes.

c) Valorar los aspectos que contribuyen en mayor medida al desarrollo inicial de la IPD en este colectivo.

\subsection{Descripción de la muestra}

La muestra está formada por 335 alumnos y alumnas, que representan a la mayor parte de la población de estudiantes de todas las especialidades que se imparten en el Máster FPEs de la Universidad de Córdoba correspondientes a las promociones 2009-10 (40,4\%) y 2010-11 (59,6\%), de los que el 53,7\% son mujeres y el resto son hombres. Esta muestra se distribuye en cuatro macroáreas de 
ROCÍO SERRANO RODRÍGUEZ Y ALFONSO PONTES PEDRAJAS

EL DESARROLLO DE LA IDENTIDAD PROFESIONAL DOCENTE EN LA FORMACIÓN INICIAL DEL PROFESORADO DE SECUNDARIA

conocimiento: Ciencias Experimentales (31,5\%), Tecnología, Dibujo y Matemáticas (30\%), Arte y Humanidades (18,1\%) y Ciencias Jurídicas y Sociales (20,4\%).

Los participantes respondieron a las cuestiones planteadas voluntariamente y mostraron una actitud colaborativa en su realización, ya que tales cuestiones formaban parte del programa-guía de actividades de aula, contando con la colaboración de un número amplio de profesores y profesoras de todas las especialidades del máster, que participaron en un proyecto de innovación docente coordinado por los autores de esta investigación. La mayoría relativa de la muestra está formada por jóvenes con una edad comprendida entre 20 y 25 años (44,5\%). Algo más de una tercera parte de la muestra tienen una edad comprendida entre $26-30$ años $(35,8 \%)$ y el resto tiene 30 años o más.

\subsection{Características del cuestionario}

Para recoger las opiniones sobre el proceso de construcción de la IPD hemos elaborado un cuestionario, incluido en el programa de actividades de aula, formado por un conjunto de preguntas abiertas sobre cuestiones relacionadas con el desarrollo de la IPD. Mediante un sistema de validación interjueces constituido por 4 expertos que compartían docencia en el Máster FPES (profesoras y profesores doctores de la Universidad de Córdoba), el cuestionario compuesto por 7 preguntas quedó reducido a 5 interrogantes, mejorando la claridad y formulación de los mismos.

Antes de su edición definitiva y una vez modificado en base a la opinión de los expertos se seleccionó una pequeña muestra (14 sujetos) y se aplicó el cuestionario para comprobar que se adaptaba a los objetivos que pretendía la investigación, realizando un estudio piloto de carácter exploratorio (Serrano et al., 2012). Después de esta aplicación, el cuestionario definitivo quedó compuesto por una batería de cinco cuestiones abiertas que se recogen en el Cuadro 1. Además de tales cuestiones se incluye una sección inicial destinada a recoger datos sociodemográficos de los y las participantes.

\subsection{Proceso de análisis de datos}

La administración del instrumento se realizó al inicio del módulo específico en la asignatura Complementos de Formación disciplinar, dentro del bloque sobre Profesionalidad docente, donde se abordan contenidos relacionados con el perfil profesional y el acceso a la función docente.

Para el análisis de las respuestas obtenidas se utilizó el programa informático Atlas.ti. Posteriormente, los datos y frecuencias obtenidas del análisis cualitativo se exportaron al paquete estadístico spss aplicando procedimientos estadísticos descriptivos (frecuencias y valores medios). 
ROCÍO SERRANO RODRÍGUEZ Y ALFONSO PONTES PEDRAJAS

EL DESARROLLO DE LA IDENTIDAD PROFESIONAL DOCENTE EN LA FORMACIÓN INICIAL DEL PROFESORADO DE SECUNDARIA

CUADRO 1

Enunciado de las preguntas que integran el cuestionario sobre IPD

1. ¿Qué crees que significa el término «identidad profesional docente» o cuáles serían las principales características de dicha identidad?

2. ¿Es igual la identidad profesional de un maestro de primaria, de un profesor de secundaria o de un docente universitario? SÍ _ No ¿Por qué?

3. ¿Crees que los futuros docentes de enseñanza secundaria desarrollan durante su carrera universitaria una identidad profesional equivalente a la que desarrollan los médicos, arquitectos, abogados, ingenieros, periodistas o cualquier otra profesión que requiera una formación universitaria? Sí $\mathrm{No}$ ¿Por qué?

4. ¿En qué etapa de su formación o de su vida profesional crees que un profesor de secundaria comienza a desarrollar un proceso de identificación con la profesión docente? ¿Por qué?

5. Señala qué aspectos crees que pueden contribuir en mayor medida a desarrollar la profesionalidad docente durante el proceso de formación inicial del profesorado de secundaria.

Fuente: Elaboración propia

\section{Resultados}

A continuación se expone el proceso de categorización generado y el resultado de frecuencias y porcentajes en cada una de las cinco preguntas que conforman nuestro instrumento.

\section{1. (Q1) ¿Qué crees que significa el término «identidad profesional docente»o cuáles serían las principales características de dicha identidad?}

La expresión de los datos descriptivos relacionados con el término IPD está marcada por la creación de dos categorías centrales: definición y perfil docente. A su vez, la primera de las categorías está compuesta por dos subcategorías: profesión y autoconciencia. También observamos que la segunda categoría se encuentra compuesta por dos subcategorías: características formativas y características personales.

A continuación se ejemplifican algunos de los relatos de los participantes en relación a estas dos categorías:

1. Definición. Profesión. Capacidad de enseñar: «Interés por trasmitir y por conseguir que un grupo de personas adquieran y comprendan un determinado conocimiento. Es de esta forma cuando el profesor se siente identificado con lo que está haciendo...».

2. Perfil docente. Características formativas. Formación general en educación: «Un profesional que se dedique a la enseñanza debe reunir otra formación 
ROCÍO SERRANO RODRÍGUEZ Y ALFONSO PONTES PEDRAJAS

EL DESARROLLO DE LA IDENTIDAD PROFESIONAL DOCENTE EN LA FORMACIÓN INICIAL DEL PROFESORADO DE SECUNDARIA

educativa que no solo esté centrada en la propia materia, es necesario que tenga una formación general en metodología, aspectos educativos, recursos...".

En la Tabla 1 se recogen las frecuencias y porcentajes de las categorías, subcategorías y códigos generados en torno a la definición de IPD. La suma de los totales parciales de los porcentajes correspondientes a las diferentes categorías es superior a cien porque la definición de IPD que hace cada persona puede incluir varios matices diferentes.

TABLA 1

Opiniones referidas a la definición de IPD

\begin{tabular}{|c|c|c|c|c|}
\hline CATEGORÍA & SUBCATEGORÍA & SuBTIPO (CÓDIGO DE ANÁLISIS) & FRECUENCIA & $(\%)$ \\
\hline \multirow{6}{*}{ DEFINICIÓN } & \multirow{2}{*}{ PROFESIÓN } & Identificación como docente & 142 & 42,4 \\
\hline & & Capacidad de enseñar & 86 & 25,7 \\
\hline & \multicolumn{2}{|r|}{ Total Parcial } & 228 & 68,1 \\
\hline & \multirow{2}{*}{ AUTOCONCIENCIA } & Aceptación social y de sí mismo & 101 & 30,1 \\
\hline & & Vocación & 57 & 17,0 \\
\hline & \multicolumn{2}{|r|}{ Total Parcial } & 58 & 47,1 \\
\hline \multirow{9}{*}{$\begin{array}{l}\text { PERFIL } \\
\text { DOCENTE }\end{array}$} & \multirow{2}{*}{$\begin{array}{c}\text { CARACTERÍSTICAS } \\
\text { FORMATIVAS } \\
\end{array}$} & Formación general en educación & 105 & 31,3 \\
\hline & & Formación específica & 30 & 8,9 \\
\hline & \multicolumn{2}{|r|}{ Total Parcial } & 35 & 40,2 \\
\hline & \multirow{5}{*}{$\begin{array}{l}\text { CARACTERÍSTICAS } \\
\text { PERSONALES }\end{array}$} & Buen comunicador & 29 & 8,7 \\
\hline & & Adaptación a cambios & 26 & 7,8 \\
\hline & & Capacidad de trabajo en equipo & 22 & 6,6 \\
\hline & & Interés por el alumnado & 21 & 6,3 \\
\hline & & Motivación & 19 & 5,7 \\
\hline & & Total Parcial & 117 & 35,1 \\
\hline
\end{tabular}

Fuente: Elaboración propia.

Alrededor de dos tercios de la muestra encuestada relaciona la definición de IPD con el ejercicio de la profesión docente (68,1\%), vinculando este concepto con la pertenencia (en el futuro) al colectivo del profesorado de secundaria $(29,9 \%)$ y destacando de forma específica la capacidad de enseñar (25,7\%). Así mismo, un conjunto importante de participantes relacionan la IPD con el proceso que supone tomar conciencia de la problemática y las características de la docencia en educación secundaria (47,1\%). Este proceso conlleva la necesidad de desarrollar una imagen positiva de la labor docente $(30,1 \%)$ y fomentar el interés vocacional por esta profesión (17\%).

En la segunda categoría de respuestas a esta cuestión se recogen opiniones que eluden a la definición de IPD y se centran más bien en caracterizar el perfil profesional que consideran más adecuado para ejercer la docencia. En primer 
ROCÍO SERRANO RODRÍGUEZ Y ALFONSO PONTES PEDRAJAS

EL DESARROLLO DE LA IDENTIDAD PROFESIONAL DOCENTE EN LA FORMACIÓN INICIAL DEL PROFESORADO DE SECUNDARIA

lugar, observamos una parte importante de respuestas que relacionan este perfil profesional con la necesidad de adquirir una formación docente de carácter general $(31,3 \%)$ y específica $(8,9 \%)$ para enseñar en centros de educación secundaria. En segundo lugar, se citan una serie de características personales como la capacidad de comunicación oral $(8,7 \%)$, de trabajar en equipo $(6,6 \%)$ y de adaptación a los cambios $(7,8 \%)$. En esta subcategoría también se incluyen cualidades como la motivación por la enseñanza $(5,7 \%)$ o el interés por el alumnado $(6,3 \%)$.

\section{2. (Q2) ¿Es igual la identidad profesional de un maestro o maestra de primaria, de un profesor o profesora de secundaria o de un docente universitario? Sí o no. ¿Por qué?}

En esta segunda pregunta, nos interesaba hacer reflexionar al alumnado tras un primer acercamiento al término IPD, con la finalidad de conocer si existen o confluyen distintas identidades en función del nivel educativo en el que nos posicionemos como docentes. El 86\% del alumnado encuestado considera que existen diferencias en la construcción de la IPD entre los profesores y las profesoras de diferentes etapas educativas. Las opiniones recogidas se han agrupado en varias categorías y subcategorías que se muestran en la Tabla 2. A continuación se citan algunos de los relatos más representativos de tales categorías.

(1) Características internas:

(1.1) Diferentes necesidades educativas para el alumnado de las distintas etapas: "... cada docente ha recibido una formación académica para dirigir su acción pedagógica a un determinado colectivo de alumnos de una etapa formativa concreta y con unas características intelectuales, culturales, psicológicas y fisiológicas concretas».

(1.2) La formación específica del profesorado de cada etapa: "Porque los maestros forjan esa identidad durante toda su carrera universitaria, y el resto lo hacen al terminar los estudios y decidirse por trabajar en la enseñanza secundaria o la docencia universitaria».

(2) Características externas:

(2.1) Planificación de la enseñanza en diferentes niveles educativos: "Se requieren competencias distintas y se usan diferentes metodologías en los distintos niveles educativos». 
ROCÍO SERRANO RODRÍGUEZ Y ALFONSO PONTES PEDRAJAS

EL DESARROLLO DE LA IDENTIDAD PROFESIONAL DOCENTE EN LA FORMACIÓN INICIAL DEL PROFESORADO DE SECUNDARIA

TABLA 2

Opiniones referidas a la IPD del profesorado de las distintas etapas educativas

\begin{tabular}{|c|c|c|c|c|}
\hline CATEGORÍA & SUBCATEGORÍA & SuBTIPO (CÓDIGO DE ANÁLISIS) & FRECUENCIA & $(\%)$ \\
\hline \multirow{7}{*}{$\begin{array}{l}\text { CARACTERÍSTICAS } \\
\text { INTERNAS }\end{array}$} & \multirow{2}{*}{ ALUMNADO } & $\begin{array}{l}\text { Necesidades del alumnado } \\
\text { diferentes }\end{array}$ & 145 & 43,3 \\
\hline & & $\begin{array}{l}\text { Problemas del alumnado } \\
\text { diferentes }\end{array}$ & 52 & 15,5 \\
\hline & \multicolumn{2}{|r|}{ Total Parcial } & 197 & 58,9 \\
\hline & \multirow{3}{*}{ PROFESORADO } & Formación específica & 55 & 16,4 \\
\hline & & Roles del profesorado & 46 & 13,7 \\
\hline & & Vocación & 18 & 5,4 \\
\hline & & Total Parcial & 119 & 35,5 \\
\hline \multirow{5}{*}{$\begin{array}{l}\text { CARACTERÍSTICAS } \\
\text { EXTERNAS }\end{array}$} & \multirow{4}{*}{$\begin{array}{l}\text { PLANIFICACIÓN } \\
\text { DE LA } \\
\text { ENSEÑANZA }\end{array}$} & Niveles Educativos & 79 & 23,6 \\
\hline & & Contenidos & 37 & 11,0 \\
\hline & & Didáctica & 36 & 10,8 \\
\hline & & Objetivos & 36 & 10,8 \\
\hline & & Total Parcial & 188 & 56,2 \\
\hline
\end{tabular}

Fuente: Elaboración propia.

Hay bastantes estudiantes que exponen como motivo principal de las diferencias en la IPD, entre los y las docentes de distintos niveles educativos, la existencia de necesidades educativas diferentes en el alumnado de cada nivel $(43,3 \%)$ y, como consecuencia, los distintos problemas que se pueden generar $(15,5 \%)$ en cada caso. La planificación de la enseñanza en cada uno de los niveles también parece marcar una distinción clara (23,6\%). Así mismo, hacen alusión a la formación específica del docente en cada una de las etapas educativas $(16,4 \%)$ y al desarrollo de roles diferentes $(13,7 \%)$. Existe una tendencia a manifestar una IPD distinta por cuestiones relacionadas con la impartición de contenidos (11\%), la consecución de los objetivos formativos $(10,8 \%)$ y la metodología didáctica empleada en el aula $(10,8 \%)$.

\section{3. (Q3) ¿Crees que los futuros y las futuras docentes de enseñanza secundaria desarrollan durante su carrera universitaria una identidad profesional equivalente a la que desarrollan los médicos, arquitectos, abogados, ingenieros, periodistas o cualquier otra profesión que requiera una formación universitaria? Sí o no. ¿Por qué?}

Las respuestas a esta cuestión se agrupan en dos categorías principales, referidas a la naturaleza especial de la profesión docente y la formación requerida para ejercer esta profesión. También se pueden distinguir varios tipos de ideas en cada una de estas categorías, según se muestra en la Tabla 3. La mayoría del alumnado del Máster FPEs piensa que la IPD del profesorado de enseñanza secundaria es 
ROCÍO SERRANO RODRÍGUEZ Y ALFONSO PONTES PEDRAJAS

EL DESARROLLO DE LA IDENTIDAD PROFESIONAL DOCENTE EN LA FORMACIÓN INICIAL DEL PROFESORADO DE SECUNDARIA

diferente a la de otras profesiones intelectuales (88,4\%), que también requieren una formación universitaria (médicos, periodistas...).

TABLA 3

Opiniones referidas a las diferencias de IPD en distintas profesiones

\begin{tabular}{|c|c|c|c|}
\hline CATEGORÍA & SUBTIPO (CÓDIGO DE ANÁLISIS) & FRECUENCIA & $(\%)$ \\
\hline \multirow{3}{*}{$\begin{array}{l}\text { PROFESIÓN } \\
\text { DOCENTE }\end{array}$} & Vocación & 173 & 51,6 \\
\hline & Salida Laboral & 40 & 12 \\
\hline & Total Parcial & 213 & 63,6 \\
\hline \multirow{4}{*}{$\begin{array}{l}\text { FORMACIÓN } \\
\text { UNIVERSITARIA } \\
\text { REQUERIDA }\end{array}$} & Formación Psicopedagógica & 77 & 23 \\
\hline & $\begin{array}{l}\text { Formación Didáctica Específica para la } \\
\text { Educación Secundaria }\end{array}$ & 17 & 5,1 \\
\hline & Formación en la práctica docente & 25 & 7,5 \\
\hline & Total Parcial & 119 & 35,6 \\
\hline
\end{tabular}

Fuente: Elaboración propia.

Para aclarar los códigos que se han generado (durante el proceso de análisis cualitativo que realiza el programa Atlas.ti) se muestran a continuación algunos ejemplos de opiniones correspondientes a los tipos de ideas más representativos:

(1) Profesión docente. Vocación: «Considero que en la profesión docente es esencial disfrutar con el trabajo que se desempeña, influido por ese componente vocacional».

(2) Formación requerida. Formación docente específica: «... porque para ser docente no solo necesitas tener conocimientos sino saberlos trasmitir, y para ello se necesita una formación didáctica adecuada, que no se ofrece en las carreras universitarias previas al Máster FPES».

Es destacable que más de la mitad de la muestra argumente como principal elemento diferenciador el interés vocacional por la profesión docente $(51,6 \%)$, seguido de la formación psicopedagógica (23\%) necesaria para el ejercicio de la profesión docente y la desvinculación en cuanto a la salida laboral (12\%). Los demás porcentajes no son muy representativos y muestran la dispersión de opiniones de los sujetos encuestados.

4.4. (Q4) ¿En qué etapa de su formación o de su vida profesional crees que un profesor o profesora de secundaria comienza a desarrollar un proceso de identificación con la profesión docente? ¿Por qué?

En el análisis de opiniones sobre esta cuestión se han considerado tres categorías vinculadas a momentos distintos de desarrollo de la IPD: inicial, intermedia 
ROCÍO SERRANO RODRÍGUEZ Y ALFONSO PONTES PEDRAJAS

EL DESARROLLO DE LA IDENTIDAD PROFESIONAL DOCENTE EN LA FORMACIÓN INICIAL DEL PROFESORADO DE SECUNDARIA

y final. En la Tabla 4 se muestran los subtipos de ideas y los datos cuantitativos correspondientes a tales categorías. A continuación se exponen algunos ejemplos de las opiniones más representativas.

(1) Inicial. IPD previa a la formación universitaria, relacionada con una vocación temprana: "Me ha interesado la enseñanza desde una edad temprana, porque me gustaba la actividad realizada por el maestro y recuerdo que a veces jugamos a ser maestros con nuestros hermanos».

(2) Intermedia:

(2.1) IPD generada durante el desarrollo de la carrera universitaria: "Cuando se está realizando la carrera universitaria y se estudian las materias específicas podemos comenzar a desarrollar la identidad con la profesión docente, porque tomas conciencia de la formación que estás recibiendo y crees que puedes transmitir esa formación a otros estudiantes".

(2.2) IPD ligada al desarrollo del Máster FPES: "Creo que la identificación con la profesión docente se intensifica en este Máster de Formación del Profesorado, ya que en las etapas de la formación anterior no hemos recibido ningún tipo de explicación ni de noción respecto a la docencia y la educación.

(3) Final. IPD ligada al ejercicio profesional en la enseñanza secundaria: "Considero que la identidad profesional de los docentes de enseñanza secundaria se desarrolla a través de la práctica docente posterior a la formación recibida para ser profesor».

TABLA 4

Opiniones referidas al momento en que se desarrolla la IPD

\begin{tabular}{|c|l|l|c|c|}
\hline CATEGORÍA & \multicolumn{1}{|c|}{ SUBCATEGORÍA } & \multicolumn{1}{c|}{ SUBTIPO (CÓDIGO DE ANÁLISIS) } & FRECUENCIA & $(\%)$ \\
\hline \multirow{2}{*}{ INICIAL } & $\begin{array}{l}\text { PREVIA A LA } \\
\text { UNIVERSIDAD }\end{array}$ & $\begin{array}{l}\text { Interés de tipo vocacional por la } \\
\text { docencia a edad temprana }\end{array}$ & 36 & 10,7 \\
\hline \multirow{2}{*}{ INTERMEDIA } & $\begin{array}{l}\text { FORMACIÓN } \\
\text { UNIVERSITARIA } \\
\text { ESPECÍFICA }\end{array}$ & Durante el desarrollo de la carrera & 68 & 20,4 \\
\cline { 2 - 6 } & Durante la formación en Secundaria & 49 & 14,6 \\
\hline \multirow{2}{*}{ FINAL } & $\begin{array}{l}\text { DESARROLLO } \\
\text { PROFESIONAL }\end{array}$ & $\begin{array}{l}\text { Total Parcial } \\
\text { Durante la vida profesional como }\end{array}$ & 117 & 35,0 \\
\hline
\end{tabular}

Fuente: Elaboración propia.

En el análisis de frecuencias y porcentajes sobre el momento de desarrollo de la IPD observamos como más de la mitad del alumnado encuestado opina que es durante la etapa laboral cuando se produce el desarrollo de la IPD (54,9\%). Un porcentaje considerable argumenta que este desarrollo se produce durante la 
formación universitaria específica (20,4\%), seguida de los y las estudiantes que consideran la formación recibida en el Máster FPES como el momento clave (14,6\%). En menor proporción, un conjunto de estudiantes atribuye el desarrollo de la IPD a un aspecto vocacional (10,7\%).

4.5. (Q5) Señala qué aspectos crees que pueden contribuir en mayor medida a desarrollar la profesionalidad docente durante el proceso de formación inicial del profesorado de secundaria

Tras el análisis de las opiniones del alumnado sobre esta última cuestión se han considerado dos categorías principales (factores internos y externos), que se pueden desglosar en tres subcategorías o dimensiones (personal, profesional y social), y varios subtipos de ideas en cada dimensión que se muestran en la Tabla 5.

TABLA 5

Opiniones sobre aspectos que ayudan a mejorar el desarrollo de la IPD

\begin{tabular}{|c|c|c|c|c|}
\hline CATEgoría & SUBCATEGORÍA & CÓDIGO & FRECUENCIA & $(\%)$ \\
\hline \multirow{10}{*}{ INTERNA } & \multirow{4}{*}{$\begin{array}{l}\text { DIMENSIÓN } \\
\text { PERSONAL }\end{array}$} & $\begin{array}{l}\text { Periodo de prácticas en los centros } \\
\text { educativos }\end{array}$ & 148 & 44,2 \\
\hline & & $\begin{array}{l}\text { Formación psicopedagógica y } \\
\text { didáctica }\end{array}$ & 73 & 21,8 \\
\hline & & Formación disciplinar previa recibida & 34 & 10,1 \\
\hline & & Pasión por la profesión (vocación) & 23 & 6,9 \\
\hline & \multicolumn{2}{|r|}{ Total Parcial } & 278 & 83,0 \\
\hline & \multirow{4}{*}{$\begin{array}{l}\text { DIMENSIÓN } \\
\text { PROFESIONAL }\end{array}$} & $\begin{array}{l}\text { Integración de experiencias } \\
\text { profesionales }\end{array}$ & 52 & 15,5 \\
\hline & & $\begin{array}{l}\text { El aprendizaje de nuevas } \\
\text { metodologías }\end{array}$ & 48 & 14,3 \\
\hline & & Uso de recursos didácticos variados & 30 & 9,0 \\
\hline & & $\begin{array}{l}\text { Fomentar la motivación en el } \\
\text { alumnado }\end{array}$ & 17 & 5,1 \\
\hline & \multicolumn{2}{|r|}{ Total Parcial } & 147 & 43,9 \\
\hline \multirow{3}{*}{ EXTERNA } & \multirow{2}{*}{$\begin{array}{l}\text { DIMENSIÓN } \\
\text { SOCIAL }\end{array}$} & $\begin{array}{l}\text { Reconocimiento social de la } \\
\text { profesión }\end{array}$ & 21 & 6,3 \\
\hline & & Ejercicio de la profesión docente & 18 & 5,4 \\
\hline & & Total Parcial & 39 & 11,7 \\
\hline
\end{tabular}

Fuente: Elaboración propia.

Cuando tratan de señalar los aspectos más influyentes para desarrollar la IPD durante el proceso de formación inicial del profesorado de secundaria, la 
ROCÍO SERRANO RODRÍGUEZ Y ALFONSO PONTES PEDRAJAS

EL DESARROLLO DE LA IDENTIDAD PROFESIONAL DOCENTE EN LA FORMACIÓN INICIAL DEL PROFESORADO DE SECUNDARIA

mayoría de los y las participantes en este estudio argumentan en clave interna, ya que relacionan los aspectos recogidos en la primera categoría con la toma de conciencia acerca de su influencia en el desarrollo personal y profesional docente. En menor medida se utilizan argumentos que relacionan la construcción de la IPD con factores externos (dimensión social). A continuación se muestran ejemplos de las opiniones más representativas de tales dimensiones del pensamiento de los y las participantes.

(1) Interna:

(1.1) Dimensión personal, relacionada con la experiencia desarrollada durante el periodo de prácticas docentes: "Se debería contar con más prácticas en los centros educativos. Es muy poco el tiempo de permanencia y apenas nos da tiempo de comprobar todo lo que hemos visto durante los módulos genéricos".

(1.2) Dimensión profesional, relacionada con la capacidad de desarrollar una enseñanza motivadora: "El mayor contacto con el alumnado desde el principio y la transmisión de la motivación ayudan a desarrollar la profesionalización docente».

(2) Externa:

(2.1) Dimensión social, relacionada con el reconocimiento social de la profesión: "Tomar conciencia uno mismo y ser capaz de transmitir a la sociedad la importancia de la labor docente».

Los datos mostrados en la Tabla 5 reflejan que los aspectos enmarcados en la dimensión personal del docente son los más relevantes para la construcción de la IPD entre los y las participantes en este estudio (83\%). Entre tales aspectos destaca la importancia concedida al periodo de prácticas en los centros educativos, la formación pedagógica y la formación disciplinar previa. Entre los aspectos relacionados con la dimensión profesional (43,9\%), destacan la integración de experiencias profesionales, el aprendizaje de nuevos métodos de enseñanza, el uso de recursos didácticos variados y el fomento de la motivación en el alumnado.

En menor proporción y en relación con la dimensión social $(11,7 \%)$, los sujetos encuestados vinculan la mejora de la IPD al reconocimiento y valoración de la profesión docente y al propio desarrollo y ejercicio de la profesión docente.

\section{DISCUSIÓN Y CONCLUSIONES}

Este estudio forma parte de un proyecto de mejora de la formación inicial del profesorado de secundaria, basado en la aplicación de un modelo innovador de formación docente (Korthagen, 2004; Perrenoud, 2010; Lim, 2011), donde tratamos de fomentar la reflexión y la metacognición de los participantes en torno a cuestiones relacionadas con el desarrollo de la profesionalidad docente durante el proceso formativo (Pontes, Serrano y Poyato, 2013). El análisis cualitativo de tales 
ROCÍO SERRANO RODRÍGUEZ Y ALFONSO PONTES PEDRAJAS

EL DESARROLLO DE LA IDENTIDAD PROFESIONAL DOCENTE EN LA FORMACIÓN INICIAL DEL PROFESORADO DE SECUNDARIA

ideas aporta información útil sobre el momento en el que comienza a desarrollarse la IPD, entre las futuras y futuros docentes, o sobre los aspectos que contribuyen en mayor medida a fomentar la identificación con la profesión docente.

En relación con el primer objetivo de este estudio los datos recogidos indican que el alumnado del Máster FPES presenta una IPD poco elaborada al comenzar el proceso de formación inicial, detectándose diferencias notables en las concepciones previas sobre la IPD de tales estudiantes, que se han expuesto al analizar los resultados cualitativos y cuantitativos de las tres primeras cuestiones. Casi la mitad del alumnado encuestado da prioridad a la necesidad de adquirir una adecuada formación en psicopedagogía y didáctica específica, además de poseer o desarrollar unas cualidades personales que les habiliten para ser buenos comunicadores (Pontes, Serrano y Poyato, 2013; Ibarras, 2014). Los participantes muestran interés por el alumnado, y destacan la necesidad de tener una buena capacidad de trabajo en equipo. También resaltan la importancia de la motivación por la docencia, como elemento clave de la IPD, a diferencia de los resultados de otros estudios previos sobre el tema (Torres, 2010), aunque puede deberse a que aún no han iniciado el contacto con la vida laboral como docentes, porque la desmotivación puede surgir al enfrentarse diariamente a los problemas de la práctica docente en centros de enseñanza secundaria (Bolívar, 2006).

Por otro lado, el alumnado del Máster FPES piensa que existen diferencias en las concepciones sobre la IPD entre profesores y profesoras de diferentes niveles educativos, porque existen unas características internas y externas que marcan la diferencia. Tanto el alumnado como el o la docente de los distintos niveles educativos presentan unas necesidades, problemas, roles y formación específica que los diferencian unos de otros. Así mismo, casi dos tercios del alumnado del Máster FPES opina que la propia planificación de la enseñanza (objetivos, contenidos, metodología, etc.) es una de las características externas al docente y al alumnado que marca una IPD diferente en cada uno de los niveles educativos. Estos resultados son similares a los obtenidos en las investigaciones de Martín, Conde y Mayor (2014).

También debemos resaltar, en relación al primer objetivo, que más de la mitad de los participantes en esta investigación creen que la vocación docente es el elemento diferenciador en la construcción de la IPD del futuro o futura docente de secundaria en relación a otras profesiones. Sin embargo, cuando definen el término IPD la vocación no resulta ser uno de los elementos más destacable siguiendo la línea de otras investigaciones precedentes (García y Martínez, 2001; Córdoba et al., 2009; Pontes et al., 2011). También destacan como elemento diferenciador la formación psicopedagógica necesaria para la práctica docente (Camacho y Padrón, 2006; Pérez, 2010).

Con relación al segundo objetivo de este estudio, observamos que las ideas de los y las participantes sobre el inicio del desarrollo de la IPD permiten diferenciar tres momentos o fases: inicial, intermedia y final. En esta investigación constatamos que la mayoría de nuestros alumnos y alumnas $(54,9 \%$ ) dan más importancia a la etapa final, es decir, el contacto con la experiencia profesional. Este hecho ya se 
ha puesto antes de manifiesto por diversos autores como Nías (1989) o Korthagen (2004), que consideran que el desarrollo de la IPD cobra verdadero sentido durante el ejercicio de la práctica docente en centros educativos. En nuestro estudio también observamos que una parte de los participantes (35,0\%) conceden importancia al desarrollo de la IPD durante la fase intermedia, es decir, durante los estudios universitarios de grado y durante el Máster FPES, por tratarse de una etapa en la que hay que decidir el futuro profesional. Se va adquiriendo conciencia de la problemática de la profesión docente y de la formación necesaria para su ejercicio, como se manifiesta en los estudios de Flores y Day (2006), Marcelo y Vaillant (2009), Hong (2010), Cabrera y Córdoba (2011) y Gewerc (2012). Es destacable que la vocación temprana no parezca tener una influencia relevante en el desarrollo inicial de la IPD, como ya manifestaron Sepúlveda y Rivas (2000).

Finalmente, en relación al tercer objetivo de esta investigación, observamos que el alumnado del Máster FPEs realiza una distinción similar a la que han encontrado anteriormente otros autores (Larraín, 2003; Bolívar, 2006) a la hora de valorar los aspectos que contribuyen en mayor medida al desarrollo inicial de la IPD entre aspirantes a ser profesores y profesoras de enseñanza secundaria. Hemos observado que la mayoría de nuestros alumnos y alumnas argumentan en clave interna relacionando el desarrollo de la IPD con un proceso de toma de conciencia que afecta al desarrollo personal. En esta categoría se concede gran importancia al periodo de prácticas docentes desarrolladas en el Máster FPES (López y Blázquez, 2012), como principal elemento que puede influir en la identidad profesional de los futuros y las futuras docentes, ya que supone el primer encuentro formal con la realidad educativa de las aulas de secundaria (Valle y Manso, 2010). También conceden importancia a la formación psicopedagógica y didáctica como elemento que favorece de manera complementaria el desarrollo de la IPD (Camacho y Padrón, 2006). En esta categoría también se recogen ideas más explícitamente relacionadas con la dimensión profesional y que aluden a la integración de experiencias profesionales reales durante la formación que les permitan conocer mejor la realidad educativa, manejar recursos didácticos variados, fomentar la motivación del alumnado y el aprender a usar nuevas metodologías de enseñanza (Márquez, 2009). Por último, hay un número menor de participantes que relacionan la construcción de la IPD con factores externos, donde apuntan hacia una dimensión social como es el reconocimiento de la actividad docente por parte del alumnado de secundaria, las familias y la sociedad en general (Esteve, 2009; Segovia y Barrero, 2012; Lobato et al., 2012).

En general, podemos indicar que los participantes de este estudio presentan una visión algo difusa del proceso de construcción de la IPD. Las circunstancias personales, los conocimientos e ideas previas con las que acceden al Máster FPEs, las experiencias previas como estudiantes y los modelos pedagógicos que han conocido influyen en la configuración de la imagen de sí mismos como futuros enseñantes. De esta manera, constatamos que la identidad profesional que acompaña al docente, y que éste va construyendo poco a poco, está estrechamente ligada y condicionada por su identidad personal y su formación (Ávalos et al., 2010). 
ROCÍO SERRANO RODRÍGUEZ Y ALFONSO PONTES PEDRAJAS

EL DESARROLLO DE LA IDENTIDAD PROFESIONAL DOCENTE EN LA FORMACIÓN INICIAL DEL PROFESORADO DE SECUNDARIA

Pensamos que los resultados obtenidos en este estudio pueden ser consecuencia, en parte, del modelo de formación inicial docente consecutivo por el que se ha optado actualmente (Maldonado, 2010), que concede prioridad a la formación específica previa sobre la formación de carácter pedagógico necesaria para la docencia. Este hecho no facilita precisamente el desarrollo de la IPD, porque muchos de los estudiantes del máster seguirán identificándose con la especialidad que han cursado durante la carrera (Esteve, 2009; Marcelo, 2009) y, sólo después, si no hay otra salida profesional, tratarán de aprender a ser profesor de secundaria (Córdoba et al., 2009). Creemos que estos resultados aportan datos interesantes para avanzar en el desarrollo de la investigación educativa sobre la construcción de la Identidad Profesional Docente (Esteve, 1997; Beijaard, Meijer y Verloop, 2004; Day, 2005), que hasta ahora se había centrado principalmente en el estudio de la IPD en profesores de secundaria en activo (Bolívar, 2006).

Con la implantación del nuevo Máster FPEs se inicia una etapa diferente que ofrece importantes oportunidades formativas para los profesores y las profesoras de secundaria, pero, también, se aprecian dificultades que habrá que ir resolviendo paulatinamente (Serrano, 2013). Pensamos que el citado máster ofrece un contexto adecuado para extender las investigaciones sobre la IPD a la formación inicial de los futuros y de las futuras docentes (Esteve, 2009).

\section{REFERENCIAS BIBLIOGRÁFICAS}

Álvarez, C. y San Fabián, J. L. (2013). Perspectivas para comprender la relación entre la teoría y la práctica en la formación del profesorado. Enseñanza \& Teaching: Revista Interuniversitaria de Didáctica, 31 (19), 23-42.

Ávalos, B.; Cavada, P.; Pardo, M. y Sotomayor, C. (2010). La profesión docente: Temas y discusiones en la literatura internacional. Estudios Pedagógicos, 36 (1), 235-263.

Ávalos, B. y Sotomayor, C. (2012). Cómo ven su identidad los docentes chilenos. Perspectiva Educacional, 51 (1), 77-95.

Beijaard, D.; Meijer, P. C. y Verloop, N. (2004). Reconsidering research on teachers professional identity. Teaching and Teacher Education, 20 (2), 107-128.

Bolívar, A. (2006). La identidad profesional del profesorado de secundaria: Crisis y reconstrucción. Archidona: Aljibe.

Bolívar, A. (2009). La gestión integrada e interactiva. En A. Romero (Ed.). Claves para mejorar la escuela secundaria. Buenos Aires: Novedades Educativas.

Bolívar, A.; Fernández, M. y Molina, E. (2005). Investigar la identidad profesional del profesorado: Una triangulación secuencia. Forum: Qualitative Social Research, 6 (1), 129-141.

Briggs, A. (2007). Exploring professional identities: middle leadership in further education colleges. School Leadership and Management, 27 (5), 471-485.

Cabrera, L. y Córdoba, M. (2011). Evaluación de un procedimiento de validación y acreditación de competencias profesionales. Revista de Investigación en Educación, 9 (2), 51-75.

Camacho, H. y Padrón, M. (2006). Malestar docente y formación inicial del profesorado: percepciones del alumnado. Revista Interuniversitaria de Formación del Profesorado, 20 (2), 209-230. 
ROCÍO SERRANO RODRÍGUEZ Y ALFONSO PONTES PEDRAJAS

EL DESARROLLO DE LA IDENTIDAD PROFESIONAL DOCENTE EN LA FORMACIÓN INICIAL DEL PROFESORADO DE SECUNDARIA

Córdoba, F.; Ortega R. y Pontes, A. (2009). Universitarios de ciencias ante la docencia en educación secundaria como expectativa profesional. Revista de Educación, 348, 401421.

Danielewicz, J. (2001). Teaching selves. Identity, pedagogy and teacher education. Albany, NY: The State of New York Press.

Day, C. (2005). Pasión por enseñar. La identidad personal y profesional del docente y sus valores. Madrid: Narcea.

Day, C. y Gu, Q. (2012). Profesores: vidas nuevas, verdades antiguas. Madrid: Narcea.

Esteve, J. M. (1997). La formación inicial de los profesores de secundaria. Una reflexión sobre el curso de cualificación pedagógica. Barcelona: Ariel Educación.

Esteve, J. M. (2009). La formación de profesores: bases teóricas para el desarrollo de programas de formación inicial. Revista de Educación, 350, 15-29.

Flores, M. y Day, C. (2006). Contexts wich shape and reshape new teacher' identities: A multi-perspective study. Teaching and Teacher Education, 22 (2), 219-232.

García, S. y Martínez, C. (2001). Las ideas de los alumnos del CAP, punto de referencia para reflexionar sobre formación docente. Revista Interuniversitaria de Formación del Profesorado, 40, 97-110.

Gewerc, A. (2011). Identidades docentes en contextos turbulentos. Espacios, tiempos y afectos. En C. Monereo y J. I. Pozo (Eds.). La identidad en Psicología de la educación (pp. 189-212). Madrid: Narcea.

Hernández, L. y Hernández, C. (2011). Hacia un modelo dinámico y eficiente de formación del profesorado. Revista Electrónica Interuniversitaria de Formación del Profesorado, 14 (1), 53-66.

Hong, J. (2010). Pre-service and beginning teachers' professional identity and its relation to dropping out of the profession. Teaching and Teacher education, 26, 1530-1543.

Ibarras, G. (2014). Ética e identidad docente. En J. M. Sancho. Aprender a ser docente en un mundo de cambio. Simposio internacional. Barcelona: UB.

Kagan, D. M. (1992). Professional growth among preservice and beginning teachers. Review of Educational Research, 62, 129-169.

Korthagen, F. (2004). In search of the essence of a good teacher: towards a more holistic approach in teacher education. Teaching and Teacher Education, 20 (1), 77-97.

Korthagen, F. (2010). Teacher reflection: What it is and what it does. The Purposes, Practices, and Professionalism of Teacher Reflectivity: Insights for Twenty-first-century. En Teachers and Students (pp. 377-401). Lanham, ML: Rowman y Littlefield.

Larraín, J. (2003). El concepto de identidad. Revista Famecos, 21, 30-42.

Lim, H.W. (2011). Concept Maps of Korean EFL Student Teachers' Autobiographical Reflections on Their Professional Identity Formation. Teaching and Teacher Education: An International Journal of Research and Studies, 27 (6), 969-981.

Lobato, C.; Fernández, I.; Garmendia, M. y Pérez, U. (2012). ¿Se puede construir la identidad del profesorado en la universidad? En Actas del VII Congreso Internacional de Docencia universitaria e innovación. Barcelona: UB-UPC.

López, H. A. y Blázquez, F. (2012). La práctica pedagógica en la formación inicial de profesores de primer ciclo de enseñanza básica en Portugal. Enseñanza \& Teaching: Revista Interuniversitaria de Didáctica, 30 (1), 23-43.

Maldonado, A. F. (2010). Problemas y Reflexiones sobre el Máster de Formación de Profesorado: Una mirada crítica desde las ciencias de la educación hacia la gobernanza universitaria. En I. González (Ed.). El nuevo profesor de secundaria. Barcelona: Graó. 
ROCÍO SERRANO RODRÍGUEZ Y ALFONSO PONTES PEDRAJAS

EL DESARROLLO DE LA IDENTIDAD PROFESIONAL DOCENTE EN LA FORMACIÓN INICIAL DEL PROFESORADO DE SECUNDARIA

Marcelo, C. (2009). Desenvolvimiento profesional docente: pasado y futuro. Revista de Ciencias de la Educación, 348, 9-22.

Marcelo, C. y Vaillant, D. (2009). Desarrollo Profesional Docente. ¿Cómo se aprende a ensenar? Madrid: Narcea.

Márquez, A. C. (2009). La formación inicial para el nuevo perfil del docente de Secundaria. Relación entre teoría y práctica. Tesis doctoral. Universidad de Málaga.

Martín, A.; Conde, J. y Mayor, C. (2014). La identidad profesional docente del profesorado novel universitario. Revista de Docencia Universitaria, 12 (4), 141-160.

Mosquera, C. J. (2011). La investigación sobre la formación de profesores desde la perspectiva del cambio didáctico. Magis. Revista Internacional de Investigación Educativa, 3 (6), 265-282.

Nias, J. (1989). Primary teachers talking: A study of teaching as work. London: Routledge.

OCDE (2012). Panorama de la Educación. Indicadores de la OCDE 2012. Madrid: Ministerio de Educación, Cultura y Deporte.

Pérez, A. I. (2010). Reinventar la profesión docente. Nuevas perspectivas y escenarios en la era de la formación y de la incertidumbre. Revista Interuniversitaria de Formación del Profesorado, 68 (2), 17-36.

Perrenoud, Ph. (2010). Desarrollar la práctica reflexiva en el oficio de enseñar: profesionalización y razón pedagógica. Barcelona: Graó.

Pontes, A.; Ariza, L. y Del Rey, R. (2010). Identidad profesional docente en aspirantes a profesorado de enseñanza secundaria. Psychology, Society, E Education, 2 (2), 131-142.

Pontes, A.; Ariza, L.; Serrano, R. y Sánchez, F. J. (2011). Interés por la docencia entre aspirantes a profesores de Ciencia y Tecnología al comenzar el proceso de formación inicial. Revista Eureka sobre Enseñanza y Divulgación de las Ciencias, 8 (2), 180-195.

Pontes, A. y Serrano, R. (2010). La formación inicial en un contexto de cambio. En I. González. El nuevo profesor de secundaria. La formación docente en el marco del Espacio Europeo de Educación Superior. Barcelona: Graó.

Pontes, A.; Serrano, R. y Poyato, F. J. (2013). Concepciones y motivaciones sobre el desarrollo profesional docente en la formación inicial del profesorado de enseñanza secundaria. Revista Eureka sobre Enseñanza y Divulgación de las Ciencias, 10 (n. ${ }^{\circ}$ Extra), 533-551.

Segovia, J. D. y Barrero, B. (2012). Reconstrucción de la identidad profesional del profesorado. Una mirada desde la orientación y la dirección. Perspectiva Educacional, 51 (2), 91-118.

Sepúlveda, M. ${ }^{a}$ P. y Rivas, J. I. (2000). Biografías profesionales. Elementos Auxiliares de Clase, 70. Universidad de Málaga.

Serrano, R. (2013). Identidad profesional, necesidades formativas y desarrollo de competencias docentes en la Formación Inicial del Profesorado de Secundaria. Tesis doctoral. Universidad de Córdoba.

Serrano, R.; Pontes, A. y Muñoz, J. M. (2012). Estudio exploratorio de la identidad profesional docente entre aspirantes a profesores de ciencias de educación secundaria en el proceso de formación inicial. En P. Membiela et al. (Eds.). Experiencias de investigación e innovación en la enseñanza de las ciencias (pp. 353-358). Ourense: Educación Editora.

Sweitzer, V. (2008). Networking to develop a professional identity: a look at the first semester experience of doctoral students in business. New Directions for Teaching and Learning, 113, 43-56. 
ROCÍO SERRANO RODRÍGUEZ Y ALFONSO PONTES PEDRAJAS

EL DESARROLLO DE LA IDENTIDAD PROFESIONAL DOCENTE EN LA FORMACIÓN INICIAL DEL PROFESORADO DE SECUNDARIA

Torres, J. (2010). Análisis del grado de satisfacción del profesorado de educación secundaria en el desarrollo de su labor docente. Contextos Educativos, 13, 27-41.

Valle, J. M. y Manso, J. (2010). La nueva formación inicial del profesorado de Educación Secundaria: modelo para la selección de buenos centros de prácticas. Revista de Educación, 354, 267-290.

Veiravé, D.; Ojeda, M.; Núñez, C. y Delgado, P. (2006). La construcción de la identidad de los profesores de enseñanza media. Biografías de profesores. Revista Iberoamericana de Educación, 40 (3), 1-13.

Zabalza, M. Á. (2012). Las competencias en la formación del profesorado: de la teoría a las propuestas prácticas. Tendencias Pedagógicas, 20, 5-32. 
